\title{
Some anisotropy indices for the characterization of Brownian textures and their application to breast images.
}

\author{
Frédéric J.P. Richard \\ Aix Marseille Université, CNRS, Centrale Marseille, I2M, UMR 7373, 13453 Marseille, \\ France.
}

\begin{abstract}
In this paper, we focus on the analysis of image textures modeled by anisotropic fractional Brownian fields. We propose some anisotropy indices intended to characterize the anisotropy of these textures. The construction of these indices relies upon the use of multi-oriented quadratic variations. It is based on directional quantities appearing in their asymptotic expectation. We show that anisotropy indices are invariant to some image transforms, and estimable from observed data. Eventually, we use anisotropy indices in combination with a measure of texture roughness to detect lesions in mammograms.
\end{abstract}

Keywords: Texture analysis, texture classification, anisotropy, roughness, fractional Brownian field, lesion detection, mammography, breast cancer.

2010 MSC: 00-01, 99-00

\section{Introduction}

Brownian textures refer to a large class of irregular and non-stationary image textures which are described by Gaussian random field models derived from the fractional Brownian motion [1]. The most famous ones are fractional Brownian 5 fields. These fields have a single parameter, the so-called Hurst index, which is directly related to a degree of texture roughness (see Section 2 for details).

Email address: frederic.richard@univ-amu.fr (Frédéric J.P. Richard)

URL: http://www.i2m.univ-amu.fr/r̃ichard/ (Frédéric J.P. Richard)

Preprint submitted to Spatial Statistics

February 11, 2016 
More generic fields include multifractional Brownian fields [2, 3] and their extensions [4, 5, 6] which can model textures with an inhomogeneous roughness. These fields have a functional Hurst index which locally quantifies the degree of roughness. Another family of models cover anisotropic Gaussian fields (e.g. anisotropic fractional Brownian fields (AFBF) [7], operator scaling Gaussian random fields [8], etc.) which are well-suited for the modeling of rough textures with directional properties. Such models have features (e.g. scalar or functional parameters) that encode directional properties of fields and may enrich the description of their texture irregularity. In this paper, we deal with the characterization and estimation of the directional heterogeneity (i.e anisotropy) of these fields.

In [9, 10, semi-parametric methods were proposed for the estimation of the anisotropy of irregular stationary fields. In [11, another method was developed 20 for the estimation of the single anisotropy parameter of a specific operator scaling random field [1]. This method is an implementation of the characterization of anisotropic Besov space by hyperbolic wavelet transforms [12. In this paper, we propose some original features, called anisotropy indices, which aims at characterizing the field anisotropy within a framework of extended AFBF (see 25 [13] and Section 2).

For the definition of these indices, we set some requirements. First, indices should represent an intrinsic quantity which is invariant to some image transforms such as rotations, rescalings, or linear changes of intensities. Second, indices should be estimable from an observed image. This second requirement 30 is probably the most stringent. Indeed, as opposed to multifractional Brownian fields whose Hurst index can be efficiently estimated using quadratic variations 14, 15, 6, 16, 17, AFBF still raise open estimation issues. In [18, a method was proposed for the estimation of their directional parameters. But, due to the use of the Radon transform, it can only be applied in a few directions with a 35 limited precision. By contrast, it is possible to accurately estimate some directional quantities which are indirectly related to parameters of AFBF. In [13, such quantities were brought out during the construction of isotropy tests (see 
Section 2 for details). They are attached to the use of multi-oriented quadratic variations, and accurately estimated using linear regression techniques. In this paper, we use these quantities as a basis for the construction of our anisotropy indices.

Fractional Brownian fields and related models known as $1 / \mathrm{f}$ models are widely spread in image processing applications, especially for the analysis of textures of mammograms (radiographic images of the breast) [19, 20, 21, 22,

45 23, 24, 25, 26, 27, 18, 28. Indeed, the Hurst index, as well as the parameter of a $1 / \mathrm{f}$ model, is directly related to the more popular fractal dimension. This quantity has been of interest for the study of lesion detectability [20, 23, 25], the detection of lesions [19, 22, 28, 26, 27, and the assessment of the breast cancer risk [21, 24]. However, it is an isotropic measure of roughness which does not account for directional properties of textures. Hence, we propose to combine such a measure with anisotropy indices so as to enrich the characterization of mammogram textures. We then test the benefit of this extended description in the context of lesion detection.

\section{Image model}

There are different ways to define image textures. In this work, textures are viewed as a random aspect of an image. To account for this randomness, we regard each image as a realization of a random field. Moreover, we focus on rough textures. Accordingly, we consider that a texture is a visual effect of an irregularity of the underlying field. This implies that textures are related to highest frequency properties of the field, and that they differ from other low-frequency aspects such as trends. The anisotropy we are interested is a feature of the image texture. Hence, it only concerns field high-frequencies. In this section, we present a random field framework which is appropriate for the stochastic modelling of trended images with rough and anisotropic textures. 
We shall be able to deal with images having large trends. Hence, we can not assume that the random field underlying an image is second-order stationary. Still, some stationary assumptions are required to infer properties of the random field from a single realization (i.e. an image). Hence, we make a weak assumption that only field increments of a certain order are stationary. In geostatistical terms, we assume that random fields are intrinsic [29, 30]:

Definition 1. Let $M \in \mathbb{N}$, and $Z$ a random field. An increment field of order $M$ of $Z$ is a random field defined, for any $y \in \mathbb{R}^{2}$, as

$$
V(y)=\sum_{i=1}^{m} \lambda_{i} Z\left(x_{i}+y\right),
$$

with some sets $\left(\lambda_{i}\right)_{i=1}^{m}$ of scalar values and $\left(x_{i}\right)_{i=1}^{m}$ of points in $\mathbb{R}^{2}$ satisfying the condition

$$
\sum_{i=1}^{m} \lambda_{i} x_{i}^{l}=0, \forall l \in \mathbb{N}^{2}, l_{1}+l_{2} \leq M .
$$

A field is intrinsic of order $M$ (or $M$-IRF) if its increment fields $V$ of order $M$ are zero mean, and second-order stationary, i.e. for any $y, \mathbb{E}(V(y))=0$, and, for any $y, z, \mathbb{E}(V(y) V(z))$ only depends on $y-z$.

75 An $M$-IRF can have a random polynomial trend of degree $M$. Hence, it can account for large non-stationary trends observed in images. Let us notice that a stationary random field can be seen as a special IRF of order $M=-1$. Indeed, when $M=-1$, Condition (1) is void so that any linear combination of $Z$ has to be stationary. Intrinsic field of order 0 correspond to fields with stationary increments. They include the isotropic or anisotropic fractional Brownian field (see [7, 18] and Section 2.2 for a definition), or the operator scaling Gaussian field [8].

Continuous $M$-IRFs are characterized by the so-called generalized covariances. These covariances are functions $K$ for which

$$
\mathbb{E}\left(Z_{\lambda, x} Z_{\mu, y}\right)=\sum_{i=1}^{m} \sum_{j=1}^{n} \lambda_{i} \mu_{j} K\left(x_{i}-y_{j}\right)
$$


holds for any pair of $M$-increments $Z_{\lambda, x}$ and $Z_{\mu, y}$ of $Z$. They have a spectral representation [29, 31, 30, 13, which extends the Bochner representation of stationary field covariances. For a large class of $M$-IRF, this representation is characterized by a spectral density $f$.

Definition 2. A M-IRF has a spectral density if its generalized covariances are of the form

$$
K(h)=\frac{1}{(2 \pi)^{d}} \int_{\mathbb{R}^{d}}\left(\cos (\langle w, h\rangle)-\mathbf{1}_{B}(w) P_{M}(\langle w, h\rangle)\right) f(w) d w+Q(h),
$$

where $P_{M}(t)=1-\frac{t^{2}}{2}+\cdots+\frac{(-1)^{M}}{(2 M) !} t^{2 M}, \mathbf{1}_{B}(w)$ is the indicator function of an arbitrary neighborhood of $0, Q$ an arbitrary even polynomial of degree $\leq 2 M$, and $f$ is an even and positive function satisfying the integrability conditions

$$
\forall A>0, \int_{|w|<A}|w|^{2 M+2} f(w) d w<\infty \text { and } \int_{|w|>A} f(w) d w<\infty .
$$

Conditions in (4) are related to field properties at low and high frequencies, respectively. In particular, as the parameter $M$ increases, the first condition becomes weaker, allowing the order of polynomial trends to be higher.

\subsection{Texture anisotropy}

In [7, some anisotropic extensions of the fractional Brownian field were defined from the spectral representation of Gaussian fields with stationary increments. These fields are IRF of order 0 characterized by a spectral density of the form

$$
g_{\tau, \eta}(w)=\tau(\arg (w))|w|^{-2 \eta(\arg (w))-2},
$$

for some even, positive, bounded, and $\pi$-periodic functions $\tau$ and $\eta$, where $\eta(s) \in$ $(H, \bar{H})$ with $0<H \leq \bar{H}<1$. The anisotropy of this model is due to the socalled topothesy function $\tau$ and Hurst function $\eta$ which are both dependent on spectral direction $\arg (w)$. In particular cases when these functions are constant, 95 fields are isotropic and correspond to fractional Brownian fields. Following [13, these anisotropic fields can be extended to IRF of an arbitrary order by only imposing $0<H<1$ and letting the upper bound $\bar{H}$ of $\eta$ varies in $(H,+\infty)$. 

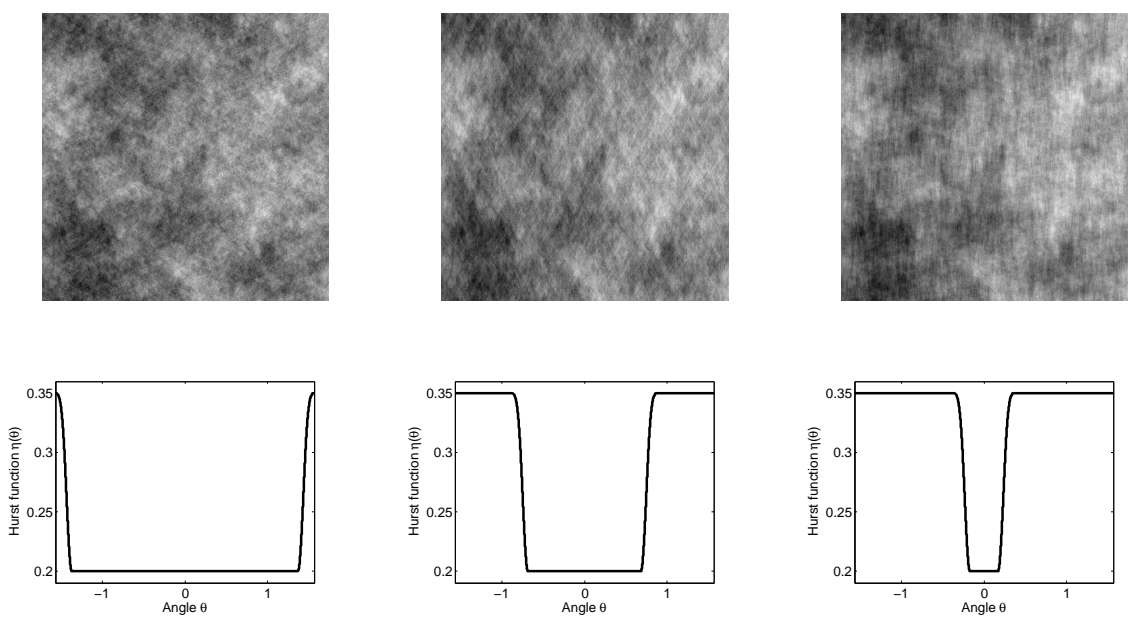

Figure 1: Some realizations of anisotropic fractional Brownian fields simulated using the turning-band method 32 . Second row: the Hurst function of these fields.

On Figure 1, we show some realizations of anisotropic fractional Brownian fields. Their topothesy functions are constant and their Hurst functions are minimal over an interval $E_{f}$ of decreasing length (from left to right, $l\left(E_{f}\right)=$ $\left.2.75, l\left(E_{f}\right)=1.37, l\left(E_{f}\right)=0.34\right)$. As observed on Figure 1, the decrease of the length of $E_{f}$ enhances the anisotropy of simulated textures.

The form of the spectral density of an AFBF is identical at all frequencies. As a consequence, properties of trends and textures of an AFBF cannot be dissociated. For instance, an AFBF cannot describe images with anisotropic textures and isotropic trends. Hence, following [13], we will make the assumption that images are sampled from an $M$-IRF whose spectral density $f$ fulfills the condition

$$
|w|>A \Rightarrow f(w)-g_{\tau_{f}, \eta_{f}}(w) \leq C|w|^{-2 H_{f}-2-\gamma},
$$

for some positive constants $A, C$ and $\gamma$, a spectral density $g_{\tau_{f}, \eta_{f}}$ of the form (5) defined with some topothesy and Hurst functions $\tau_{f}$ and $\eta_{f}$, and $H_{f}=$ $\operatorname{ess}_{\inf } \eta_{f}(s)$. As it only concerns high-frequencies of the field, this condition does not constrain image trends. It only implies that image textures are similar 
to those of anisotropic fractional Brownian fields.

In this context, we will say that a texture is isotropic if the topothesy and Hurst functions $\tau_{f}$ and $\eta_{f}$ of the field underlying images are both almost everybe quantified from 0 to 1 by $1-H$.

Besides, the Hölder irregularity of a $M$-IRF can be characterized from the asymptotic behavior of its spectral density at high-frequencies [33, 13]. Let $Z$ be a field with a density $f$ satisfying Condition (6). Further assume that

$$
E_{f}=\left\{s \in[0, \pi), \eta_{f}(s)=H_{f}, \tau_{f}(s) \neq 0\right\} .
$$

is a subset of $[0, \pi)$ of positive measure. Then, according to Proposition 2.5 of [13, the field $Z$ is $H_{f}$-Hölder. Hence, by imposing Conditions (6) and (8), we restrict the domain of our analysis to irregular images with rough textures. Let 


\section{Anisotropy indices}

This section is devoted to anisotropy indices. We first present the multioriented quadratic variations which are at the basis of their construction. We then define them and state some of their properties. Eventually, we deal with their estimation.

Throughout the section, we will assume that an image is a realization of a random field $Z$ on a grid $\llbracket 1, N \rrbracket^{2}$. We denote by $Z^{N}[m]=Z(m / N)$ the image intensity at position $m \in \mathbb{Z}^{2}$.

\subsection{Background}

Multi-oriented quadratic variations were introduced in [13] to construct isotropy tests. The definition of these variations is based on the computation of image increments, which give information about image variations at highest observed scales. Furthermore, these increments are not only computed at different scales but also in different orientations. This is done using transforms $T_{u}$ defined for a given $u \in \mathbb{Z}^{2} \backslash\{(0,0)\}$ as a composition of a rotation of angle $\arg (u)$ and a rescaling of factor $|u|$

$$
T_{u}=\left(\begin{array}{rr}
u_{1} & -u_{2} \\
u_{2} & u_{1}
\end{array}\right)=|u|\left(\begin{array}{rr}
\cos (\arg (u)) & -\sin (\arg (u)) \\
\sin (\arg (u)) & \cos (\arg (u))
\end{array}\right),
$$

Increments in direction $\arg (u)$ at scale $|u|$ are obtained by a discrete convolution

$$
\forall m \in \mathbb{Z}^{d}, V_{u}^{N}[m]=\sum_{k \in \mathbb{Z}^{2}} v[k] Z^{N}\left[m-T_{u} k\right],
$$

with an appropriate convolution kernel $v$. This kernel is chosen so as to ensure that the convolution annihilates any polynomial of a predefined order $K$ (kernel of order $K$ ). Some kernels selected in [13] for their optimality are given for $L \in \mathbb{N} \backslash\{0\}$ by

$$
v\left[l_{1}, l_{2}\right]=(-1)^{l_{1}}\left(\begin{array}{l}
L \\
l_{1}
\end{array}\right),
$$

${ }_{135}$ if $\left(l_{1}, l_{2}\right) \in \llbracket 0, L \rrbracket \times\{0\}$ and 0 otherwise; $\left(\begin{array}{l}n \\ k\end{array}\right)$ stands for the binomial coefficient. Such a kernel is of order $K=L-1$. 
The information provided by increments are summarized into a single random variable called quadratic variation

$$
W_{u}^{N}=\frac{1}{N_{e}} \sum_{m \in \mathcal{E}_{N}}\left(V_{u}^{N}[m]\right)^{2},
$$

where $\mathcal{E}_{N}$ is a set of cardinal $N_{e}$ containing positions $m$ where increments can be computed on grid points. To get information at different scales and orientations, we compute quadratic variations for different vectors $u$ indexed in a set $\mathcal{I}$. For any $k \in \mathcal{I}$, we also compute normalized log-variations

$$
\tilde{W}_{u_{k}}^{N}=\log \left(W_{u_{k}}^{N}\right)-\sum_{m \in \mathcal{I}} \lambda_{m} \log \left(W_{u_{m}}^{N}\right)
$$

for some positive weights $\lambda_{k}$ that satisfy $\sum_{k \in \mathcal{I}} \lambda_{k}=1$. We gather all these variations into a single random vector $Y^{N}=\left(\tilde{W}_{u_{k}}^{N}\right)_{k \in \mathcal{I}}$. Following [13] (Theorem 3.4), we can specify the asymptotic probability distribution of this random vector $Y^{N}$.

Theorem 1. Assume that the random field $Z$ is a continuous Gaussian $M$ IRF with a spectral density $f$ satisfying Condition (6). Further assume that the Hurst index $H_{f}$ belongs to $(0,1)$ and the set $E_{f}$ defined by Equation (8) is of positive measure. Consider a log-variation vector $Y^{N}$ constructed using a kernel $v$ of order $K>M$. For all $k \in \mathcal{I}$, define random variables $\epsilon_{k}^{N}$ such that

$$
Y_{k}^{N}=H_{f} x_{k}+\beta_{f, v}\left(\arg \left(u_{k}\right)\right)+\epsilon_{k}^{N},
$$

where $x_{k}=\log \left(\left|u_{k}\right|^{2}\right)-\sum_{m \in \mathcal{I}} \lambda_{m} \log \left(\left|u_{m}\right|^{2}\right)$ and

$$
\beta_{f, v}\left(\arg \left(u_{k}\right)\right)=\mathcal{C}_{f, v}\left(\arg \left(u_{k}\right)\right)-\sum_{m \in \mathcal{I}} \lambda_{m} \mathcal{C}_{f, v}\left(\arg \left(u_{m}\right)\right),
$$

with

$$
\mathcal{C}_{f, v}(\theta)=\log \left(\frac{1}{(2 \pi)^{2}} \int_{E_{f}} \tau_{f}(\varphi) \int_{\mathbb{R}^{+}}|\hat{v}(\rho \vec{u}(\varphi-\theta))|^{2} \rho^{-2 H_{f}-1} d \rho d \varphi\right),
$$

$\hat{v}$ the discrete Fourier transform of $v$, and $\vec{u}(\varphi)=(\cos (\varphi), \sin (\varphi))^{\prime}$.

Then, as $N$ tends to $+\infty$, the random vector $\left(N \epsilon_{k}^{N}\right)_{k \in \mathcal{I}}$ tends in distribution to a centered Gaussian vector. 
Interestingly, Theorem 1 brings out some information that quadratic vari-

145

a linear relationship between variations $Y^{N}$ and some parameters $H_{f}$ and $\beta_{f, v}$ depending both on the spectral density of the field. The Hurst index $H_{f}$ is the parameter which directly characterizes the field irregularity. Intercepts $\beta_{f, v}$ carry a directional information which will be used to define anisotropic indices regression procedure which will be presented in Section 3.3 .

\subsection{Definition and properties}

Intercepts $\beta_{f, v}$ which appears in Equation (14) of Theorem 1 has a noticeable property: they vanish when the field texture is isotropic, and differ when it is not. This property is ensured by the following proposition (see Appendix A for a proof).

Proposition 1. Take the same conditions as in Theorem 1. Then, $\mathcal{C}_{f, v}$ is constant over $[0, \pi)$ if and only if the field is isotropic.

Besides, for appropriate weights $\left(\lambda_{k}\right)_{k}$ and a fixed $\theta$, the intercept $\beta_{f, v}(\theta)$

approximate the difference between the value of the function $\mathcal{C}_{f, v}$ at $\theta$ and its integral over $[0, \pi)$. These remarks lead us to the definition of anisotropic indices.

Definition 4 (Anisotropy indices). Take the same conditions as in Theorem 1. Let $p$ be in $(0,+\infty]$, and $\|\cdot\|_{p}$ be a norm (or pseudo-norm) of $L^{p}([0, \pi) ; \mathbb{R})$. The p-anisotropy index $A_{p, v}(f)$ of an intrinsic random field whose spectral density $f$ fulfills Condition (6) is defined as

$$
A_{p, v}(f)=\left\|\mathcal{C}_{f, v}(\cdot)-\frac{1}{\pi} \int_{0}^{\pi} \mathcal{C}_{f, v}(\varphi) d \varphi\right\|_{p}
$$

where $\mathcal{C}_{f, v}$ is defined by Equation (15). In particular, for $p=1,2,+\infty$, it is 
defined as

$$
\begin{aligned}
A_{1, v}(f) & =\frac{1}{\pi} \int_{0}^{\pi}\left|\mathcal{C}_{f, v}(\theta)-\frac{1}{\pi} \int_{0}^{\pi} \mathcal{C}_{f, v}(\varphi) d \varphi\right| d \theta \\
A_{2, v}(f) & =\frac{1}{\pi} \int_{0}^{\pi}\left(\mathcal{C}_{f, v}(\theta)-\frac{1}{\pi} \int_{0}^{\pi} \mathcal{C}_{f, v}(\varphi) d \varphi\right)^{2} d \theta \\
A_{\infty, v}(f) & =\sup _{\theta \in[0, \pi)}\left|\mathcal{C}_{f, v}(\theta)-\frac{1}{\pi} \int_{0}^{\pi} \mathcal{C}_{f, v}(\varphi) d \varphi\right|
\end{aligned}
$$

respectively.

ॠThe anisotropic index has some important properties, which are shown in Appendix A

Proposition 2. Take the same conditions as in Theorem 1, and set $p \in] 0,+\infty]$. Then,

(i) $A_{p, v}(f)=0$ if and only if the field texture is isotropic.

(ii) For any positive scalars $\alpha, \lambda$, and rotation matrix $R_{\psi}$ of angle $\psi$,

$$
A_{p, v}\left(\alpha f \circ\left(\lambda R_{\psi}\right)\right)=A_{p, v}(f)
$$

Property (i) ensures that the anisotropic index has a minimal value 0 when the fields are isotropic, and that the minimum is exclusively reached by isotropic fields. Property (ii) implies that the anisotropic index is invariant to rotations 170 and rescalings of the image, and linear transforms of its intensities.

We get some other interesting properties in cases when the kernel $v$ is monodirectional, i.e. if $v\left[k_{1}, k_{2}\right]=v_{1}\left[k_{1}\right] v_{2}\left[k_{2}\right]$ and $v_{2}\left[k_{2}\right]=0$ whenever $k_{2} \neq 0$ (see Equation (10) for examples).

Proposition 3. Take the same conditions as in Theorem 1, and set $p \in(0,+\infty]$.

(i) For any mono-directional kernel $v$ of order $K>M$, we have

$$
\begin{gathered}
A_{p, v}(f)=\tilde{A}_{p}(f), \text { where } \tilde{A}_{p}(f)=\left\|\tilde{\mathcal{C}}_{f}(\cdot)-\frac{1}{\pi} \int_{0}^{\pi} \tilde{\mathcal{C}}_{f}(\varphi) d \varphi\right\|_{p}, \\
\text { and } \tilde{\mathcal{C}}_{f}(\theta)=\log \left(\int_{E_{f}} \tau_{f}(\varphi)|\cos (\theta-\varphi)|^{2 H_{f}} d \varphi\right)
\end{gathered}
$$




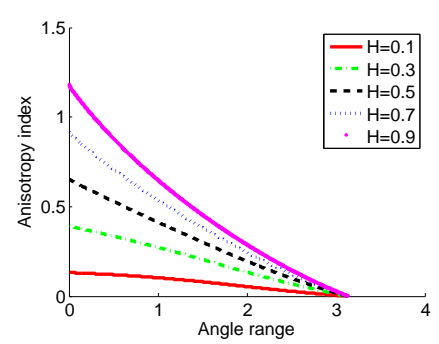

(a)

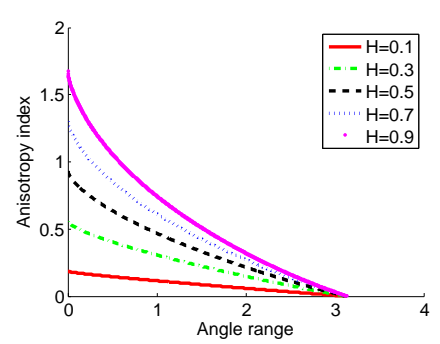

(b)

Figure 2: Theoretical values of the anisotropy indices for elementary anisotropic fractional Brownian fields: (a) $A_{1}$, (b) $A_{2}$

(ii) If we further assume that $E_{f}=\left(\alpha_{1}, \alpha_{2}\right)$ for $-\pi / 2 \leq \alpha_{1}<\alpha_{2} \leq \pi / 2$, and that $\tau_{f}$ is almost everywhere constant on $E_{f}$, then $A_{p, v}(f)$ is of the form (20) with a function $\tilde{\mathcal{C}}_{f}(\theta)$ which is the logarithm of the function

$$
\begin{cases}\mathcal{B}_{H}\left(\frac{1-\sin \left(\theta+l\left(E_{f}\right)\right)}{2}\right)+\mathcal{B}_{H}\left(\frac{1-\sin (\theta)}{2}\right) & \text { if } \pi / 2-l\left(E_{f}\right) \leq \theta \leq \pi / 2 \\ \mathcal{B}_{H}\left(\frac{1+\sin \left(\theta+l\left(E_{f}\right)\right)}{2}\right)+\mathcal{B}_{H}\left(\frac{1+\sin (\theta)}{2}\right) & \text { if }-\pi / 2-l\left(E_{f}\right) \leq \theta \leq-\pi / 2 \\ \left|\mathcal{B}_{H}\left(\frac{1-\sin \left(\theta+l\left(E_{f}\right)\right)}{2}\right)-\mathcal{B}_{H}\left(\frac{1-\sin (\theta)}{2}\right)\right| & \text { otherwise }\end{cases}
$$

where $l\left(E_{f}\right)=\alpha_{2}-\alpha_{1}$ and $\mathcal{B}_{H}$ is the incomplete Beta function given by

$$
\forall t \in[0,1], \mathcal{B}_{H}(t)=\int_{0}^{t} u^{H-1 / 2}(1-u)^{H-1 / 2} d u .
$$

Property (i) ensures that the value of the anisotropic index does not depend on the choice of the increment kernel $v$ (when restricting to mono-directional kernels). Property (ii) gives a computable formula of the anisotropic index of the so-called elementary fractional Brownian field whose topothesy function is constant on the set $E_{f}$ and null outside.

Using Formula 22, we computed anisotropy indices of fields shown in Figure 1. from left to right, we got $A_{2}=0.03, A_{2}=0.17, A_{2}=0.30$. Hence, we observed that the anisotropy enhancement is associated to an increase of anisotropy indices. 
To further illustrate this association, we computed the anisotropy indices of elementary fractional Brownian fields, i.e. fields whose topothesy function is constant on the set $E_{f}$ and null outside. On Figure 2, we plotted values of anisotropy indices as a function of the length $l\left(E_{f}\right)$ for different minimal Hurst index $H_{f}$. We can observe that a decrease of the length $l\left(E_{f}\right)$ of $E_{f}$ produces an increase of anisotropy indices. This suggests that anisotropy indices could quantify some degrees of anisotropy.

\subsection{Estimation}

For the ease of presentation, we will use a multi-index $k=(i, j)$ to identify terms involved in the linear model (13). The first index $i$ will refer to an ith angle $\varphi_{i}$ in a set $\left\{\arg \left(u_{k}\right), k \in \mathcal{I}\right\}$ of size $m$, and the index $j$ to an jth rescaling factor in a set $\left\{\left|a_{k}\right|, \arg \left(u_{k}\right)=\varphi_{i}\right\}$ of size $n_{i}$. Angles $\varphi_{i}$ are arranged in $(-\pi / 2, \pi / 2]$ by increasing order. We assume that $\varphi_{m}=\pi / 2$, and set $\varphi_{0}=-\pi / 2$ by convention. We define $\alpha_{i}=\varphi_{i}-\varphi_{i-1}, \beta_{i}=\beta_{f, v}\left(\varphi_{i}\right)$, and $H=H_{f}$.

For $p \in(0,+\infty)$, we estimate the anisotropic index $A_{p}$ with

$$
\hat{A}_{p}=\left(\sum_{i=1}^{m} \sum_{j=1}^{n_{i}} \lambda_{i j}\left|\hat{\beta}_{i}\right|^{p}\right)^{(1 / p)}
$$

where variables $\hat{\beta}_{i}$ are some estimators of $\beta_{i}$, and $\lambda_{i j}$ some positive weights satisfying $\sum_{j=1}^{n_{i}} \lambda_{i j}=\frac{\alpha_{i}}{\pi}$ for all $i \in \llbracket 1, m \rrbracket\left(e . g . \quad \lambda_{i j}=\frac{\alpha_{i}}{\pi n_{i}}\right)$. For $p=+\infty$, we estimate it with

$$
\hat{A}_{\infty}=\sup _{i \in \llbracket 1, m \rrbracket}\left|\hat{\beta}_{i}\right| .
$$

Estimates $\hat{\beta}_{i}$ of $\beta_{i}$ are obtained by applying a linear regression technique to the model 13 . More precisely, they are computed by minimizing the ordinary least square criterion

$$
J\left(H, \beta_{1}, \cdots, \beta_{m}\right)=\sum_{i=1}^{m} \sum_{j=1}^{n_{i}}\left(Y_{i j}-H x_{i j}-\beta_{i}\right)^{2}
$$

under the constraint that $\sum_{i=1}^{m} \alpha_{i} \beta_{i}=0$. This leads to estimates

$$
\hat{\beta}_{i}=\left(\bar{Y}_{i \cdot}-c_{0} \frac{\alpha_{i}}{n_{i}} \sum_{k=1}^{m} \alpha_{k} \bar{Y}_{k} .\right)-\widehat{H}\left(\bar{x}_{i}-c_{0} \frac{\alpha_{i}}{n_{i}} \sum_{k=1}^{m} \alpha_{k} \bar{x}_{k} .\right),
$$


and

$$
\widehat{H}=\frac{\sum_{i=1}^{m}\left(\sum_{j=1}^{n_{i}} x_{i j} Y_{i j}-n_{i} \bar{x}_{i} \cdot \bar{Y}_{i \cdot}\right)+c_{0}\left(\sum_{i=1}^{m} \alpha_{i} \bar{x}_{i \cdot}\right)\left(\sum_{i=1}^{m} \alpha_{i} \bar{Y}_{i .}\right)}{\sum_{i=1}^{m} \sum_{j=1}^{n_{i}} x_{i j}^{2}-\sum_{i=1}^{m} n_{i} \bar{x}_{i .}^{2}+c_{0}\left(\sum_{i=1}^{m} \alpha_{i} \bar{x}_{i .}\right)^{2}}
$$

where $\bar{x}_{i}=\frac{1}{n_{i}} \sum_{j=1}^{n_{i}} x_{i j}, \bar{Y}_{i}=\frac{1}{n_{i}} \sum_{j=1}^{n_{i}} Y_{i j}$, and $c_{0}=1 / \sum_{i=1}^{m} \frac{\alpha_{i}^{2}}{n_{i}}$.

\section{Numerical study}

In this section, we present an evaluation of estimators of anisotropy indices defined in Section 3.3 .

\subsection{Estimation error}

We simulated 10000 realizations of elementary fractional Brownian fields on a square grid of length $N=200$. The Hurst function of these fields was equal to a same Hurst index $H_{f} \in(0,1)$ on an interval of the form $E_{f}=$ $\left[\phi_{0}-\delta_{0} / 2, \phi_{0}+\delta_{0} / 2\right]$. Their topothesy function was constant on $E_{f}$, and null outside. The value of $H_{f}$ determines the irregularity of the field, the value of $\phi_{0}$ its anisotropy direction, and $\delta_{0}$ its degree of anisotropy. For each simulated field, parameters $H_{f}, \phi_{0}$ and $\delta_{0}$ were sampled independently from a uniform law on $(0.05,0.95),(-\pi / 2, \pi / 2)$, and $(0.02, \pi)$, respectively.

Our evalution was focused on elementary fractional Brownian fields which only cover special cases of anisotropic fields we deal with. In general, the topothesy function of fields may vary on $E_{f}$. However, each anisotropic field can be approximated by a sum of independent elementary fractional Brownian fields. Hence, we expect our evaluation to give a fair account of estimation performances on arbitrary anisotropic fields.

On each simulated field, we computed quadratic variations of image increments in directions $\arg (u)$ and at scales $|u|$ prescribed by vectors $u$ of the set $\mathcal{U}=\{u \in \llbracket 0, N \rrbracket \times \llbracket-N, N \rrbracket, 1 \leq|u| \leq 42\}$ (see Equations (9) and (11)). To compute increments, we used a kernel $v$ of the form 110 for $L=2$.

Then, we estimated anisotropy indices $A_{1}, A_{2}$ and $A_{\infty}$ using the regression procedure described in Section 3.3 This procedure was applied several 
times to quadratic variations associated to different subsets $\mathcal{U}_{\rho}$ of $\mathcal{U}$ depending on a maximal scale $\rho$. For $\rho \in[2,42]$, subsets $\mathcal{U}_{\rho}$ were composed of all vectors $u \in \mathcal{U}$ such that $|u|<\rho$ and there exists another $v \in \mathcal{U},|v|<\rho$ and $\arg (u)=\arg (v)$. Denoting $\mathcal{I}_{\rho}$ the set of indices of vectors of $\mathcal{U}_{\rho}$ and according to Equation 23 , , estimates of anisotropic indices obtained with a subset $\mathcal{U}_{\rho}$ are $\hat{A}_{p}^{\rho}=\left(\sum_{k \in \mathcal{I}_{\rho}} \lambda_{k}\left|\hat{\beta}_{k}\right|^{p}\right)^{\frac{1}{p}}$ for $p \in(0,+\infty)$ and $\hat{A}_{\infty}^{\rho}=\sup _{k \in \mathcal{I}_{\rho}}\left|\hat{\beta}_{k}\right|$ for $p=+\infty$.

For each anisotropic index and maximal scale, we computed the mean square error as the average of square differences between theoretical and estimated values of the anisotropy index over simulations. We used Formula $(22)$ of Proposition 3 to evaluate the theoretical indices.

Estimation errors are partly due to biases induced by discrete approximations of anisotropy indices. These approximations depend on $\rho$. For $p \in$ $(0,+\infty)$, they are given by

$$
\left(\sum_{k \in \mathcal{I}_{\rho}} \lambda_{k}\left|\mathcal{C}_{f, v}\left(\arg \left(u_{k}\right)\right)-\sum_{l \in \mathcal{I}_{\rho}} \lambda_{l} \mathcal{C}_{f, v}\left(\arg \left(u_{l}\right)\right)\right|^{p}\right)^{\frac{1}{p}},
$$

where $\mathcal{C}_{f, v}$ is defined by Equation 15 . For $p=+\infty$, it is

$$
\max _{k \in \mathcal{I}_{\rho}}\left|\mathcal{C}_{f, v}\left(\arg \left(u_{k}\right)\right)-\sum_{l \in \mathcal{I}_{\rho}} \lambda_{l} \mathcal{C}_{f, v}\left(\arg \left(u_{l}\right)\right)\right|
$$

To assess the approximation effect, we computed an error (bias) as the average of square differences between theoretical and approximated values of an anisotropy index over simulations. The approximated anisotropy indices were also evaluated using Formula 22 of Proposition 3 .

Another source of error comes from the variance of quadratic variations involved in estimation. To see this variance effect, we computed another error (variance) as the average of square differences between estimated and approximated values of an anisotropy index over simulations.

On Figure 3, we plotted errors as a function of the maximal scale $\rho$. As $\rho$ was increased, there were more and more quadratic variations involved estimation. As a result, errors due to variances kept on increasing. Conversely, 


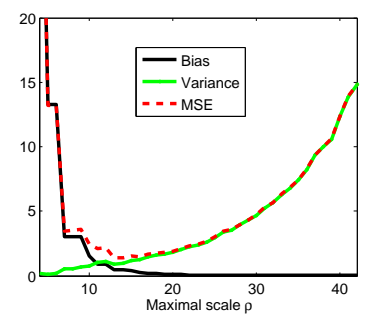

(a)

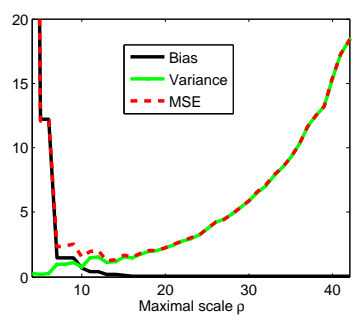

(b)

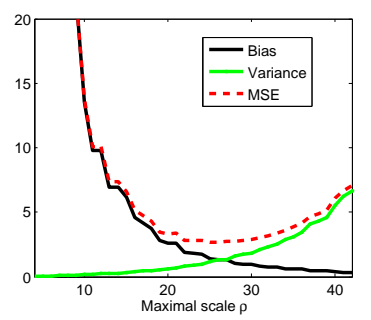

(c)

Figure 3: Relative errors for the evaluation of (a) $A_{1}$, (b) $A_{2}$ and (c) $A_{\infty}$. All errors are expressed in percent of the variance of anisotropy index values.

as $\rho$ was increased, approximation errors got lower and lower since the number of variation directions also increased. Up to some scales, improvements of approximation accuracy compensated for variance increases, and estimation errors (MSE) reduced. Above these scales, variance effects became predominant, and estimation errors started to increase. For anisotropy indices $A_{1}$ and $A_{2}$ (graphs (a) and (b)), estimation errors reached a minimum at about the scale $\rho=15$. For $A_{\infty}$ (graph (c)), the error was minimal at an higher scale $(25)$ due to a worse approximation of the index.

\subsection{Influence of simulation parameters}

We further investigated the effect of simulation parameters on the estimation of anisotropy indices. We focused on $A_{2}$ and set the maximal scale $\rho=15$.

In Table 1, we present errors with respect to ranges of different parameters. The estimation was sensitive to the field irregularity $H_{f}$. As $H_{f}$ gets close to 1 , variances of quadratic variations increases. Hence, variance errors, and a fortiori estimation errors, were larger for $H_{f}$ close to 1 than to 0 . Besides, the orientation of the anisotropy $\phi_{0}$ had a slight effect on the estimation. Approximation errors were higher for $\phi_{f}$ close to 0 and $\pi / 2$ than for directions in between. The degree of isotropy $\delta_{0}$ had also an effect on estimation. The error is particularly high when the support length $\delta_{0}$ is small, i.e. when the anisotropy is strong. 


\begin{tabular}{|c|ccc|c|ccc|c|cc|}
\hline$H_{f}$ & $E_{d}$ & $E_{v}$ & $E$ \\
\hline$(0.05,0.23)$ & 0 & 0.3 & 0.3 \\
$(0.23,0.41)$ & 0.1 & 0.9 & 1.0 \\
$(0.41,0.59)$ & 0.1 & 1.6 & 1.7 \\
$(0.59,0.77)$ & 0.2 & 2.1 & 2.2 \\
$(0.77,0.95)$ & 0.3 & 2.7 & 3.1 \\
\hline$(0,0.31)$ & 0.2 & 1.7 & 2.0 \\
$(0.31,0.63)$ & 0 & 1.4 & 1.4 \\
$(0.63,0.94)$ & 0.1 & 1.3 & 1.3 \\
$(0.94,1.26)$ & 0.1 & 1.5 & 1.5 \\
$(1.26,1.57)$ & 0.3 & 1.8 & 2.1 \\
\hline$(0.01,0.32)$ & 0.6 & 4.3 & 5.1 \\
$(0.32,0.63)$ & 0 & 1.3 & 1.4 \\
$(0.63,0.95)$ & 0 & 0.7 & 0.7 \\
$(0.95,1.26)$ & 0 & 0.6 & 0.6 \\
$(1.26,1.57)$ & 0 & 0.7 & 0.7 \\
\hline
\end{tabular}

Table 1: Analysis of errors made in computing the anisotropic index $A_{2}$ with respect to each simulation parameter. Errors denoted by $E_{d}, E_{v}$ and $E$ are the approximation bias, the variance error and the MSE, respectively. They are expressed in percent of the variance of anisotropy index values.

\section{Application to mammograms}

In this section, we present an application of anisotropy indices to the detection of lesions on mammograms.

\subsection{Context}

Breast cancer is a leading cause of woman mortality, which is particularly widespread in western countries. The early detection of this disease increases treatment possibilities and the rate of survival. Currently, mammography is recognized as the most effective imaging modality for an early detection, and used as a conventional tool for screening (see 34 for a review of epidemiological studies about mammography screening). However, there are still numerous cancers missed by mammography examination. Computer-aided detection (CAD) systems have been developed to aid radiologists in detecting lesions and try to reduce overlooked cancers (see [35, 36, 37, 38, 39 for reviews of lesion detection methods).

However, the automatic detection of lesions on mammograms (images produced by mammography) is a challenging task. Mammograms are X-ray projections of breast tissues composing breasts. Due to breast diversity and inhomogeneity, textures of these images may vary drastically not only between 

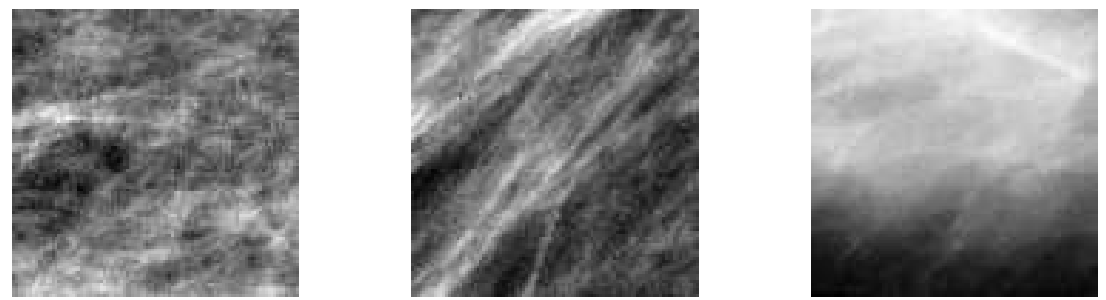

$H=0.17, A_{2}=0.22$

$H=0.34, A_{2}=0.34$

$H=0.58, A_{2}=0.69$
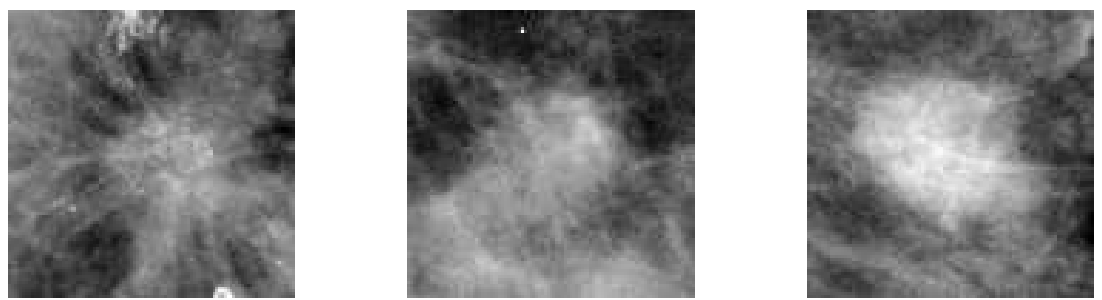

$H=0.41, A_{2}=0.17$

$H=0.65, A_{2}=0.32$

$H=0.73, A_{2}=0.22$

Figure 4: Examples of regions extracted from mammograms. Images of the first row are from normal cases. Images of the second row show lesions; from left to right, spiculated, ill-defined, and circumscribed masses.

women but also within a same breast. Such variations are illustrated on the first row of Figure 4. Mammographic patterns due to lesions are also very diverse. On the second row of Figure 4 , we show some lesions exhibiting a central mass (a bright circular area) whose shape, margin and appearance are highly variable. Some lesions may not even have a distinct mass, as it is the case with the so-called architectural distortions or asymmetries. Architectural distortions are perceived on mammograms as local distortions of the normal parenchymal texture. Such lesions are often missed by radiologists, and poorly detected by commercial and academic CAD systems [40, 38. Asymmetries may only be detectable by a comparison of bilateral mammograms 38 .

In what follows, we propose a method to detect the presence of lesions in mammograms. In this method, we use estimates of both the Hurst index and an anisotropy index to classify image regions of interest into normal and abnormal. 


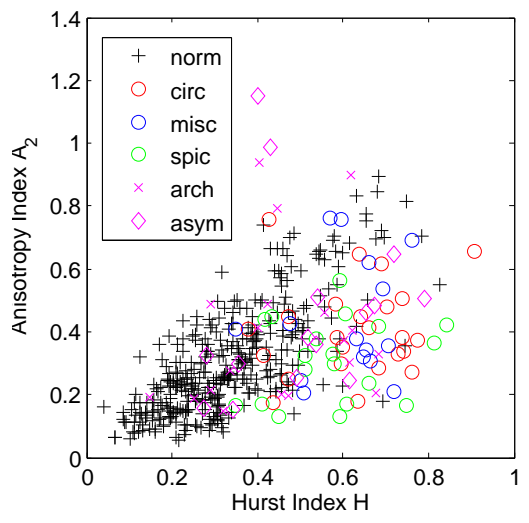

(a)

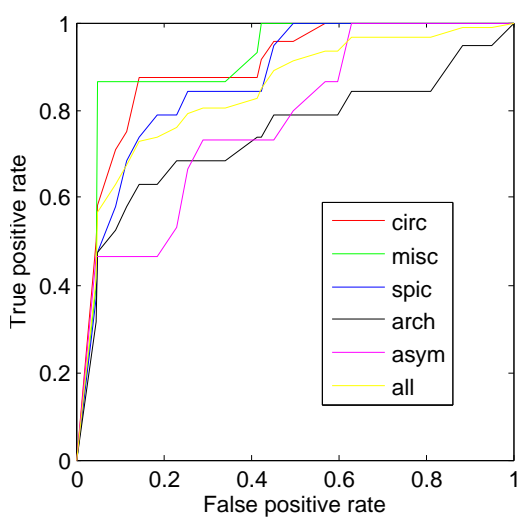

(b)

Figure 5: Classification of ROIs extracted from mammograms. Image (a): values of the Hurst and anisotropy indices $\left(\hat{H}, \hat{A}_{2}\right)$ of each ROI, (b) ROC curves obtained after a classification of ROIs.

\subsection{Lesion detection}

We applied our classification approach to the mini MIAS database [41]. In this database, we extracted regions of interest (ROI) of size $100 \times 100$ centered at the lesion barycenter within 92 pathological mammograms. We included all types of lesions except micro-calcifications. We also randomly selected 358 ROIs within normal mammograms.

On each ROI, we computed increments $V_{u}^{N}$ (see Equation 9 for vectors ${ }_{300} u \in \mathbb{Z}^{2}$ having a scale $|u|$ between $\sqrt{2}$ pixels $(0.28 \mathrm{~mm})$ and 40 pixels $(8 \mathrm{~mm})$ and an $\operatorname{argument} \arg (u)$ where at least 2 vectors $u$ had the same argument. Due to the small size of ROI, we used the kernel defined by Equation 10 with $L=1$. Since this kernel is only of order 0 , we compensated for trends in each ROI by fitting and subtracting a polynomial of order 1 to the image. For each ROI, we computed the irregularity and anisotropy indices $\hat{H}$ and $\hat{A}_{p}$ (with $p=1,2$ or $+\infty)$.

Values of these index pairs (for $p=2$ ) are shown on image (a) of Figure 5. On average, the abnormal ROIs seemed smoother than the normal ones (higher values of $H$ ). The relative smoothness of abnormal ROIs could be 

rates associated to different thresholds. Eventually, we plotted Reicever Operating Characteristic (ROC) curves (see Figure 5(b)) and computed areas under these curves (AUC) (see Table 2).

\begin{tabular}{|l|c|c|c|c|c|c|}
\hline Lesion type & circ & misc & spic & arch & asym & all \\
\hline Number of cases & 19 & 19 & 24 & 15 & 15 & 92 \\
\hline AUC (with $H$ only) & 0.895 & 0.912 & 0.861 & 0.685 & 0.771 & 0.827 \\
AUC (with $\left.\left(H, A_{2}\right)\right)$ & 0.897 & 0.916 & 0.869 & 0.743 & 0.776 & 0.843 \\
AUC (with $\left.\left(H, A_{1}\right)\right)$ & 0.88 & 0.895 & 0.855 & 0.7 & 0.778 & 0.823 \\
AUC (with $\left.\left(H, A_{\infty}\right)\right)$ & 0.812 & 0.716 & 0.891 & 0.671 & 0.63 & 0.754 \\
\hline
\end{tabular}

Table 2: The AUC of the ROC curves by lesion types.

The AUC is an indicator in $[0,1]$ which is often used in medical applica330 tions to compare detection methods. Using this criterion, the best method is the one for for which the AUC is the closest to 1. Here, the best AUC were 
obtained using the anisotropic index $A_{2}$. It reached 0.843 for all lesion types. It was particularly high $(>0.85)$ for the detection of all kinds of masses (circumscribed 'circ', ill-defined 'misc', and spiculated 'spic'). It was quite good 335 (> 0.74) for other subtle lesions (architectural distortions 'arch' and asymmetries 'asym'). Comparing the AUC obtained with and without using $A_{2}$, we noticed that information provided by $A_{2}$ greatly improves the detection of architectural distortions. However, the improvement was not as significant for other lesions.

\section{Discussion}

We defined some indices which are intended to characterize the anisotropy of image textures modeled by anisotropic fractional Brownian fields. We showed that these indices are intrinsic quantities in the sense that they are invariant to several image transforms (rotation, rescalings and linear intensity changes). We also constructed estimators of these indices, and evaluated them on synthetic data. Results showed that the estimation mean square error was lower than $2 \%$ for two selected indices $\left(A_{1}\right.$ and $\left.A_{2}\right)$.

Our anisotropy indices differ from the one proposed in [11]. In [11, the index was the parameter of a specific operator scaling random fields. Here, indices were rather non-parametric quantities which characterized a large class of anisotropic fields; due to their regularity properties [42, 43], operating scaling random fields are likely to belong to this class. In [11, the anisotropy parameter was estimated using hyperbolic wavelets. By construction, these wavelets are dependent on image axes. Hence, the wavelet analysis had to be performed on different image rotations so as to account for arbitrary anisotropy directions. As it required image interpolations, the use of rotations was likely to bias the estimation procedure. By contrast, our indices were estimated using quadratic variations computed on the image grid at several scales and orientations without any interpolation. Consequently, our estimation procedure was quite robust to the anisotropy direction. 

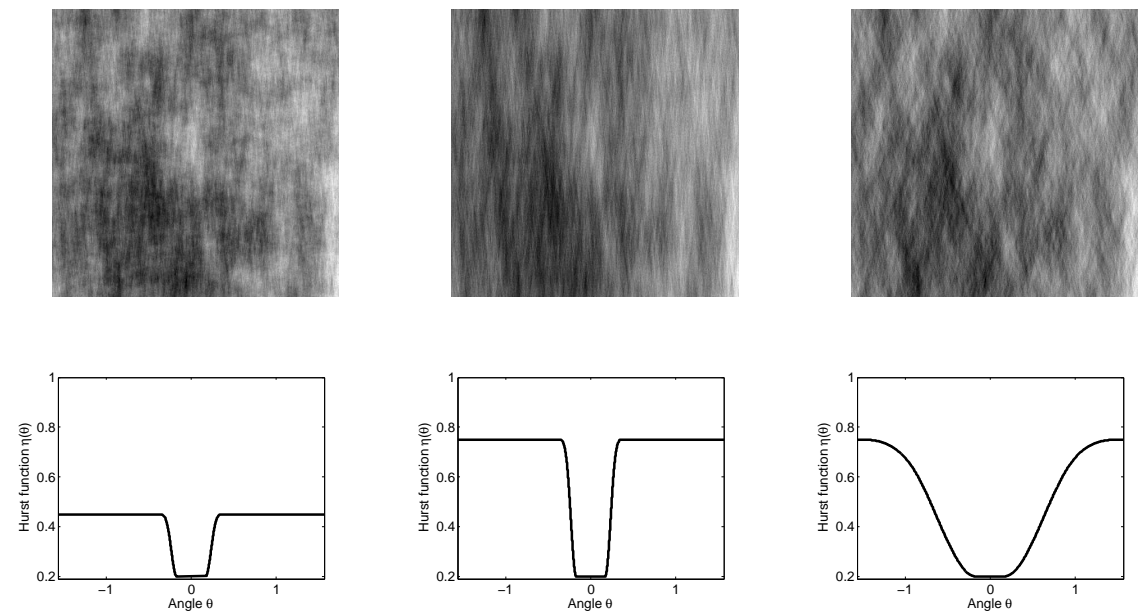

Figure 6: Some simulated fields (top row) with a same anisotropy index $A_{2}=0.30$ (first row) but different Hurst functions (second row). In all examples, the minimal value $H_{f}$ of the Hurst function and the set $E_{f}$ where it is minimal are the same.

The construction of anisotropy indices relied upon the use of multi-oriented quadratic variations introduced in [13]. More precisely, it was based on directional quantities which appear in the asymptotic expression of the expectation of these variations. These asymptotic quantities depend on the texture irregularity $H_{f}$ (the minimal value of the Hurst function $\eta_{f}$ of the observed AFBF). This dependence should be taken into account when comparing the anisotropy of textures with different degree of roughness. Moreover, asymptotic quantities only depend on values of the topothesy and Hurst functions on the set $E_{f}$ of directional frequencies where the Hurst function is minimal. Therefore, anisotropy indices can not differentiate anisotropic textures where these functions exclusively differ outside $E_{f}$. Examples of such textures are shown on Figure 6 .

We combined anisotropy indices with the Hurst index so as to describe both the irregularity and anisotropy of mammogram textures. We used this combined description to detect the presence of lesions in regions of interest. Despite the variability of mammogram textures and lesion patterns, we obtained encourag- 
ing results for all lesion types. The benefit of anisotropy indices was particularly obvious for architectural distortions which are lesions among the most difficult to detect.

\section{References}

[1] B. B. Mandelbrot, J. Van Ness, Fractional Brownian motion, fractionnal noises and applications, SIAM Rev. 10 (1968) 422-437.

[2] A. Benassi, S. Jaffard, D. Roux, Elliptic Gaussian random processes, Rev. Mathem. Iberoamericana 13 (1) (1997) 19-89.

[3] R. Peltier, J. Levy Vehel, Multifractional Brownian motion: definition and preliminary results, Tech. Rep. 2645, INRIA (1996).

[4] J. Ayache, J. Lévy-Véhel, Generalized multifractional Brownian motion: definition and preliminary results, in: L. Lévy-Véhel, Tricot (Eds.), Fractals: Theory and Applications in Engineering, Springer, 1999, pp. 17-32.

[5] J. Ayache, J. Lévy-Véhel, The generalized multifractional Brownian motion, Stat. Inference Stoch. Process. 3 (1) (2000) 7-18.

[6] A. Benassi, P. Bertrand, S. Cohen, J. Istas, Identification of the Hurst index of a step fractional Brownian motion, Stat. Inference Stoch. Process. $3(1 / 2)(2000) 101-111$.

[7] A. Bonami, A. Estrade, Anisotropic analysis of some Gaussian models, J. Fourier Anal. Appl. 9 (2003) 215-236.

[8] D. Benson, M. M. Meerschaert, B. Bäumer, H. P. Scheffler, Aquifer operator-scaling and the effect on solute mixing and dispersion, Water Resour. Res. 42 (2006) 1-18.

[9] G. Chan, T. Wood, Increment-based estimators of Fractal dimension for two-dimensional surface data, Stat. Sinica 10 (2000) 343-376. 
[10] S. Davies, P. Hall, Fractal analysis of surface roughness by using spatial data, J. Roy. Statist. Soc. Ser. B 61 (1999) 3-37.

[11] S. Roux, M. Clausel, B. Vedel, S. Jaffard, P. Abry, Self-similar anisotropic texture analysis: The hyperbolic wavelet transform contribution, IEEE Trans. Image Process. 22 (11) (2013) 4353-4363.

[12] P. Abry, M. Clausel, S. Jaffard, S. Roux, B. Vedel, Hyperbolic wavelet transform: an efficient tool for multifractal analysis of anisotropic textures, Rev. Mat. Iberoam. 31 (1) (2015) 313-348.

[13] F. Richard, Tests of isotropy for rough textures of trended images, Stat. Sinica (2016), In press, DOI:10.5705/ss.202014.0077.

[14] J. Ayache, J. Lévy-Véhel, On the identification of the pointwise Hölder exponent of the generalized multifractional Brownian motion, Stoch. Proc. Appl. 111 (1) (2004) 119-156.

[15] A. Ayache, P. Bertrand, J. Lévy Véhel, A central limit theorem for the quadratic variations of the step fractional Brownian motion, Stat. Inference Stoch. Process. 10 (2007) 1-27.

[16] A. Benassi, S. Cohen, J. Istas, Identifying the multifractional function of a Gaussian process, Statist. Probab. Lett. 39 (1998) 337-345.

${ }_{420}^{4}$ [17] J. Coeurjolly, Identification of multifractional Brownian motion, Bernoulli 11 (6) (2005) 987-1008.

[18] F. Richard, H. Biermé, Statistical tests of anisotropy for fractional brownian textures. application to full-field digital mammography, J. Math. Imaging Vis. 36 (3) (2010) 227-240.

${ }_{425}$ [19] S. Banik, R. M. Rangayyan, J. Desautels, Detection of architectural distortion in prior mammograms using fractal analysis and angular spread of power, in: SPIE Medical Imaging, International Society for Optics and Photonics, 2010, pp. 762408-762408. 
[20] A. Burgess, F. Jacobson, P. Judy, Human observer detection experiments with mammograms and power-law noise, Med. Phys. 28 (4) (2001) 419-437.

[21] C. Caldwell, S. Stapleton, D. Holdsworth, et al., Characterisation of mammographic parenchymal patterns by fractal dimension, Phys. Med. Biol. 35 (2) (1990) 235-247.

[22] Q. Guo, J. Shao, V. Ruiz, Investigation of support vector machine for the detection of architectural distortion in mammographic images, in: J. Phys.: Conf. Ser., Vol. 15, 2005, pp. 88-94.

[23] J. Heine, S. Deine, R. Velthuizen, et al., On the statistical nature of mammograms, Med. Phys. 26 (11) (1999) 2254-2269.

[24] J. Heine, P. Malhorta, Mammographic tissue, breast cancer risk, serial image analysis, and digital mammography: tissue and related risk factors, Acad. Radiol. 9 (2002) 298-316.

[25] B. Grosjean, L. Moisan, A-contrario detectability of spots in textured backgrounds, J. Math. Imaging Vis. 33 (3) (2009) 313-337.

[26] R. Rangayyan, S. Prajna, F. Ayres, J. Desautels, Detection of architectural distortion in prior screening mammograms using gabor filters, phase portraits, fractal dimension, and texture analysis, Int. J. Comput. Assist. Radiol. Surg. 2 (6) (2008) 347-361.

[27] R. Rangayyan, S. Banik, J. Desautels, Computer-aided detection of architectural distortion in prior mammograms of interval cancer, J. Digit. Imaging 23 (5) (2010) 611-631.

[28] G. Tourassi, D. Delong, C. Floyd, A study on the computerized fractal analysis of architectural distortion in screening mammograms, Phys. Med. Biol. 51 (5) (2006) 1299-1312.

[29] J. Chilès, P. Delfiner, Geostatistics: modeling spatial uncertainty, 2nd Edi455 tion, J. Wiley, 2012. 
[30] G. Matheron, The intrinsic random functions and their applications, Ad. Appl. Prob. 5 (1973) 439-468.

[31] I. Gelfand, N. Y. Vilenkin, Generalized functions, Vol. 4: Applications to harmonic analysis, Academic Press, 1964.

[32] H. Biermé, M. Moisan, F. Richard, A turning-band method for the simulation of anisotropic fractional Brownian field, J. Comput. Graph. Statist. 24 (3) (2015) 885-904.

[33] H. Biermé, Champs aléatoires : autosimilarité, anisotropie et étude directionnelle, Ph.D. thesis, University of Orleans, France (2005).

[34] M. Løberg, M. L. Lousdal, M. Bretthauer, M. Kalager, Benefits and harms of mammography screening, Breast Cancer Res. 17 (1) (2015) 63.

[35] K. Ganesan, U. Acharya, C. Chua, et al., Computer-aided breast cancer detection using mammograms: a review, IEEE Rev. Biomed. Eng. 6 (2013) 77-98.

[36] L. Mina, N. Isa, A review of computer-aided detection and diagnosis of breast cancer in digital mammography, J. Med. Sci. 15 (3) (2015) 110.

[37] A. Oliver, J. Freixenet, J. Marti, et al., A review of automatic mass detection and segmentation in mammographic images, Med. Image Anal. 14 (2) (2010) 87-110.

[38] R. Rangayyan, F. Ayres, L. Desautels, A review of computer-aided diagnosis of breast cancer: Toward the detection of subtle signs, J. Franklin Inst. 344 (3) (2007) 312-348.

[39] M. Sampat, M. Markey, A. Bovik, et al., Computer-aided detection and diagnosis in mammography, Handbook of image and video processing 2 (1) (2005) 1195-1217. 
[40] J. Baker, E. Rosen, J. Lo, et al., Computer-aided detection (CAD) in screening mammography: sensitivity of commercial CAD systems for detecting architectural distortion, Am. J. Roentgenol. 181 (4) (2003) 10831088.

[41] J. Suckling, J. Parker, D. Dance, et al., The Mammographic Image Analysis Society digital mammogram database, in: Exerpta Medica, Vol. 1069 of International Congress Series, 1994, pp. 375-378.

[42] H. Biermé, C. Lacaux, Hölder regularity for operator scaling stable random fields, Stoch. Proc. Appl. 119 (7) (2009) 2222-2248.

[43] M. Clausel, B. Vedel, An optimality results about sample paths properties of Operator Scaling Gaussian Random Fields in anisotropic Besov spaces, Annals of the University of Bucharest (mathematical series) 4 (2013) 375409.

\section{Appendix A. Proofs}

Proof of Proposition 1. According to Equation 15,,

$$
\mathcal{C}_{f, v}(\theta)=C_{0}, \forall \theta \Leftrightarrow K(\theta)=\frac{1}{\pi} \int_{0}^{\pi} \mathbf{1}_{E}(\varphi) \tau_{f}(\varphi) W_{H}(\theta-\varphi) d \varphi=\frac{C_{0}}{\pi}, \forall \theta,
$$

where $\mathbf{1}_{E}$ is the indicator function of the set $E$, and

$$
W_{H}(\varphi)=\int_{0}^{+\infty}|\hat{v}(\rho \vec{u}(-\varphi))|^{2} \rho^{-2 H-1} d \rho .
$$

But, computing the discrete Fourier transform of $K$, we have for all $n \in \mathbb{Z}^{2}$,

$$
\widehat{K}[n]=\widehat{\mathbf{1}_{E} \tau_{f}}[n] \widehat{W}_{H}[n],
$$

where $\hat{f}[n]=\frac{1}{\pi} \int_{0}^{\pi} f(\theta) e^{-i 2\langle n, \theta\rangle} d \theta$ denotes Fourier coefficients of a $\pi$-periodic function $f$. Hence,

$$
\mathcal{C}_{f, v}(\theta)=C_{0}, \forall \theta \Leftrightarrow \widehat{\mathbf{1}_{E} \tau_{f}}[n] \widehat{W}_{H}[n]=\left\{\begin{array}{ccc}
C_{0} / \pi & \text { if } & n=0 \\
0 & \text { if } & n \neq 0 .
\end{array}\right.
$$


Thus, inversing the Fourier transform, we obtain

$$
\mathcal{C}_{f, v}(\theta)=C_{0}, \forall \theta \Leftrightarrow \mathbf{1}_{E}(\theta) \tau_{f}(\theta)=\frac{C_{0}}{\pi \widehat{W}_{H}[0]},
$$

for almost all $\theta$. Moreover, integrating $K$ over $(0, \pi)$ in Equation A.1], we get

$$
\int_{E} \tau(\varphi) d \varphi \widehat{W}_{H}[0]=C_{0} .
$$

495

Given Equation A.3), this implies that the Lebesgue measure of the set $E$ is equal to $\pi$. Consequently, $\mathcal{C}_{f, v}$ is constant over $(0, \pi)$ if and only if $\tau_{f}$ is almost everywhere constant over $(0, \pi)$.

Proof of Proposition 2. Property (i) is a direct consequence of Proposition 1 and properties of a norm (or pseudo-norm).

We focus on the proof of Property (ii). Let $\alpha$, and $\lambda$ be two positive scalars, and $R_{\psi}$ a rotation matrix of angle. Set $g=\alpha f \circ\left(\lambda R_{\psi}\right)$. According to (6), we have $E_{g}=E_{f}-\psi$, and $H_{g}=H_{f}$. Furthermore, for all $\theta \in E_{g}$,

$$
\tau_{g}(\theta)=\alpha \lambda^{-2 H_{f}-1} \tau_{f}(\theta+\psi)
$$

Hence, from Equation (15), we get

$$
\mathcal{C}_{g, v}(\theta)=\log \left(\alpha \lambda^{-2 H_{f}-1}\right)+\log \left(\int_{E_{f}-\psi} \tau_{f}(\varphi+\psi) W_{H}(\theta-\varphi) d \varphi\right),
$$

where $W_{H}$ is given by Equation $\mathrm{A} .2$. Therefore, by a coordinate change $\zeta=$ $\varphi-\psi$, we obtain

$$
\mathcal{C}_{g, v}(\theta)=\log \left(\alpha \lambda^{-2 H_{f}-1}\right)+\mathcal{C}_{f, v}(\theta-\psi)
$$

Then, using Equation (16), we get

$$
\mathcal{A}_{p, v}(g)=\left\|\mathcal{C}_{f, v}(\cdot-\psi)-\frac{1}{\pi} \int_{0}^{\pi} \mathcal{C}_{f, v}(\varphi-\psi) d \varphi\right\|_{p},
$$

and, thus, $\mathcal{A}_{p, v}(g)=\mathcal{A}_{p, v}(f)$. 
Proof of Proposition 3. Let $v$ be a mono-directional filter, i.e. $v\left[k_{1}, k_{2}\right]=$ $v_{1}\left[k_{1}\right] v_{2}\left[k_{2}\right]$ and $v_{2}\left[k_{2}\right]=0$ whenever $k_{2} \neq 0$. Then,

$$
C_{f, v}(\theta)=\int_{E_{f}} \tau_{f}(\varphi) \int_{0}^{+\infty}\left|\hat{v}_{1}(\rho \cos (\theta-\varphi))\right|^{2} \rho^{-2 H_{f}-1} d \rho d \varphi
$$

Using the coordinate change $\lambda=\rho|\cos (\theta-\varphi)|$, it comes that

$$
C_{f, v}(\theta)=\Lambda\left(H_{f}, v\right) \int_{E_{f}} \tau_{f}(\varphi)|\cos (\theta-\varphi)|^{2 H_{f}} d \varphi
$$

where $\Lambda\left(H_{f}, v\right)=\int_{0}^{+\infty}\left|\hat{v}_{1}(\lambda)\right|^{2} \lambda^{-2 H_{f}-1} d \lambda$. Then, Equation 20 follows from the definition of the anisotropy index.

In the particular case when $E_{f}=\left(\alpha_{1}, \alpha_{2}\right)$ and $\tau_{f}$ is constant over $E_{f}$, we have

$$
C_{f, v}(\theta)=\lambda_{0} \int_{E_{f}}|\cos (\theta-\varphi)|^{2 H_{f}} d \varphi
$$

for some positive constant $\lambda_{0}$. The integral of this equation can be further specified using Corollary 3 of supplementary materials of 32 . This directly leads to Equation 22 . 